\title{
Improvement of Three B Spline Curve Interpolation Algorithm and Simulation
}

\author{
Wanjun Zhang ${ }^{1,2,3, a}$, Shanping Gao ${ }^{1, b}$, Xiyan Cheng ${ }^{1, c}$, Feng Zhang ${ }^{2, d}$ \\ ${ }^{1}$ Quanzhou Institute of Information Engineering, 36200, China \\ ${ }^{2}$ School of Mechanical Engineering, Xian Jiao tong University, 741049, China \\ ${ }^{3}$ Lanzhou Industry and Equipment Co, Ltd, Lanzhou 730050, China \\ agszwj_40@163.com, b358391056@qq.com, c326593363@qq.com, d116543048@qq.com
}

Keywords: Three B Spline Curve; Interpolation Arithmetic; CNC System; Simulation and Analysis.

\begin{abstract}
In order to reduce the calculation amount of Three B spline curve, combined with Three B spline curve mathematical properties. Firstly this paper introduce on the algorithm of the Three B spline curve improvement, it is the in the interpolation cycle under the condition of certain interpolation increment only and interpolation speed, by changing the interpolation increments can achieve correction curve of interpolation. Through the research on the Three B spline curve interpolation algorithm, Three B spline curve interpolation calculation and general computational complexity, proposed an improved interpolation algorithm, can reduce the time and improve the efficiency of interpolation. Then, an improved method is given by using MATLAB simulation, the results show that the interpolation algorithm can reduce interpolation computation and interpolation operation debugging time and reduce the overshoot, it has strong adaptability and versatility, provides a new method for complex dynamic control of uncertain systems.
\end{abstract}

\section{Introduction}

NURBS has been widely used in CAD/CAM thanks to its unique advantages in modeling complex components. So direct interpolation of NURBS becomes the key technique for the development of CNC machine tools, which has been studied by many scientific researchers and institutions [1].

Research on Three B spline curve interpolation algorithm has a large number of documents, many of which are in order to produce fast algorithm of curve on the computer and the design of the Three B spline curve, most of the solutions of triangular equations, parallel algorithms such as recursive coupling, cyclic reduction method, matrix decomposition method, but for multi core computer all applicable [2]. Zhao Guoyong et al. A fast and real time interpolation algorithm for NURS curve interpolation in high speed and high precision NC machining [3]. Researcher [4-8] curve parameters adaptive interpolation algorithm, improved algorithm, parallel machine tool interpolation algorithm is proposed, which improves the approximation error of Three B spline curve. The interpolation algorithm [9-21] given a curve improvement and correction curve, Taylor formula of first order in the expansion, the increment interpolation and interpolation speed, the derivative can achieve the purpose of curve interpolation. Although the above algorithm can obtain a constant speed, without considering the error control. For the interpolation algorithm of NURBS curve interpolation, Taylor's expansion first order and two order solution is more complicated, and the processing error is larger.

Finally, through the research on the Three B spline curve interpolation algorithm, NURBS curve interpolation calculation and general computational complexity, proposed an improved interpolation algorithm, can reduce the time and improve the efficiency of interpolation interpolation. Then,the improved method is given by using MATLAB simulation, the results show that the interpolation algorithm can reduce interpolation computation and interpolation operation debugging time and reduce the overshoot, it has strong adaptability and versatility, provides a new method for complex dynamic control of uncertain systems. 


\section{Improved of Three B spline curve interpolation algorithm}

\subsection{Mathematical foundation of Three B spline curve}

The Three B spline curve of pretreatment control three parameters (control points and weights, the node vector) and a feed (feed rate) as the start of the NC program, the Three B spline curve interpolator internal $\mathrm{CNC}$, servo motor drive motor shaft movements, Three B spline curve interpolation.

Three B spline curve of a piecewise rational polynomial function of the vector, the expression of [10]:

$$
p_{i}{ }^{(u)}=\mathrm{A}_{i-3} \mathrm{p}_{i-3} u^{3}+\mathrm{A}_{i-2} \mathrm{p}_{i-2} u^{2}+\mathrm{A}_{i-1} \mathrm{p}_{i-1}{ }^{u+} \mathrm{A}_{i} \mathrm{p}_{i}
$$

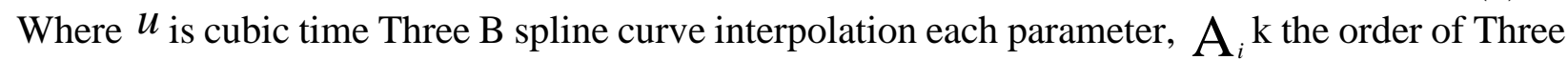
B spline curve interpolation?

\subsection{Improved three B spline curve interpolation pretreatment}

Before the interpolation calculated contour curve interpolation points for interpolation preprocessing, obtained in an interpolation period and feed speed, and the normal vector value, in order to simplify the interpolation computation and $\Delta u$ improve the real-time interpolation, $n_{x}, n_{y}, n_{z}$ will use coefficient curve to solve the vector method, the mathematical method of vector coefficient curve as follows.

$$
n=\left(p_{i}^{\prime}(u) \times p_{i}^{\prime \prime}(u)\right) \times p_{i}^{\prime}(u)=a_{4} u^{4}+a_{3} u^{3}+a_{2} u^{2}+a_{1}^{u+} a_{0}
$$

The corresponding spatial coordinate axes of the three coordinate axes

$$
\begin{aligned}
& n_{x}=a_{4 x} u^{4}+a_{3 x} u^{3}+a_{2 x} u^{2}+a_{1 x} u+a_{0 x} \\
& n_{y}=a_{4 y} u^{4}+a_{3 y} u^{3}+a_{2 y} u^{2}+a_{1 y} u+a_{0 y} \\
& n_{z}=a_{4 z} u^{4}+a_{3 z} u^{3}+a_{2 z} u^{2}+a_{1 z} u+a_{0 z}
\end{aligned}
$$

By formula (1), we can know that the trajectory vectors of the three axes in the space coordinate system are $\Delta x, \Delta y, \Delta z$, respectively, and the interpolation is designed to find out the interpolation increment of a period $x_{i}(u), y_{i}(u), z_{i}(u)$. According to the corresponding relation $V$ of the feed rate and the normal vector $\Delta z$, and the interpolation step $n_{x}, n_{y}, n_{z}$, the interpolation step is mapped to the $3 \mathrm{D}$ coordinate system, and the corresponding increment of the corresponding parameters $\Delta u$ is obtained.

$a_{4 x}, \cdots, a_{0 x}, a_{4 y}, \cdots, a_{0 y}, a_{4 z}, \cdots, a_{0 z}, n_{x}, n_{y}, n_{z}$ and $\mathrm{A}_{i-3} \mathrm{p}_{i-3}, \cdots, \mathrm{A}_{i} \mathrm{p}_{i}$ The other coefficients can be calculated separately in the pretreatment, In order to find out

$$
\left|\frac{d p_{i}^{(u)}}{d u}\right| \text { and } \frac{V \cdot T}{\left|\frac{d p_{i}^{(u)}}{d t}\right|_{u=u_{i}}}
$$

To prepare, the interpolation cycle $T$ can be reduced a lot of tedious calculation, improve the real-time interpolation algorithm.

Improvement of three B spline curve real-time interpolation algorithm

Supposed $T=t_{i+1}-t_{i}$, According to the Taylor two order expansions (7).

$$
u_{i+1}=u_{i}+\left.\frac{d u}{d t}\right|_{t=t_{i}} T+\left.\frac{1}{2} \frac{d^{2} u}{d t^{2}}\right|_{t=t_{i}} T^{2}+\text { H.O.T. }
$$

Formula H.O.T.: refers to the Taylor expansion of the higher order terms (order Terms High).

The formula (6) is expanded, and the first order expressions are obtained.

$$
u_{i+1}=u_{i}+\left.\frac{d u}{d t}\right|_{t=t_{i}}\left(t_{i+1}-t_{i}\right)
$$

As the feed rate along the curve, the curve of the time derivative obtained 


$$
V=\left\|\frac{\left.d p_{i}^{(} u\right)}{d t}\right\|=\left\|\frac{d p_{i}^{(u)}}{d u}\right\| \frac{d u}{d t}
$$

Get the first order of Taylor formula

$$
u_{i+1} \approx u_{i}+\left.\frac{V \cdot T}{\left|\frac{d p_{i}^{(}(u)}{d t}\right|}\right|_{u=u_{i}}
$$

When the interpolation period is, it can be satisfied with the first order expansion of the Taylor formula.

$$
\Delta u=\boldsymbol{u}_{i+1}-\boldsymbol{u}_{i}=\left.\frac{V \cdot T}{\left|\frac{d p_{i}^{(u)}}{d t}\right|}\right|_{u=u_{i}}
$$

By formula (11) can be seen in other conditions are given, the interpolation increment and interpolation speed, through a derivative can achieve the purpose of curve interpolation.

\section{Algorithm flow chart}

According to the process analysis of the above improved interpolation algorithm, the flow chart of the interpolation algorithm is given, as shown in figure 1.

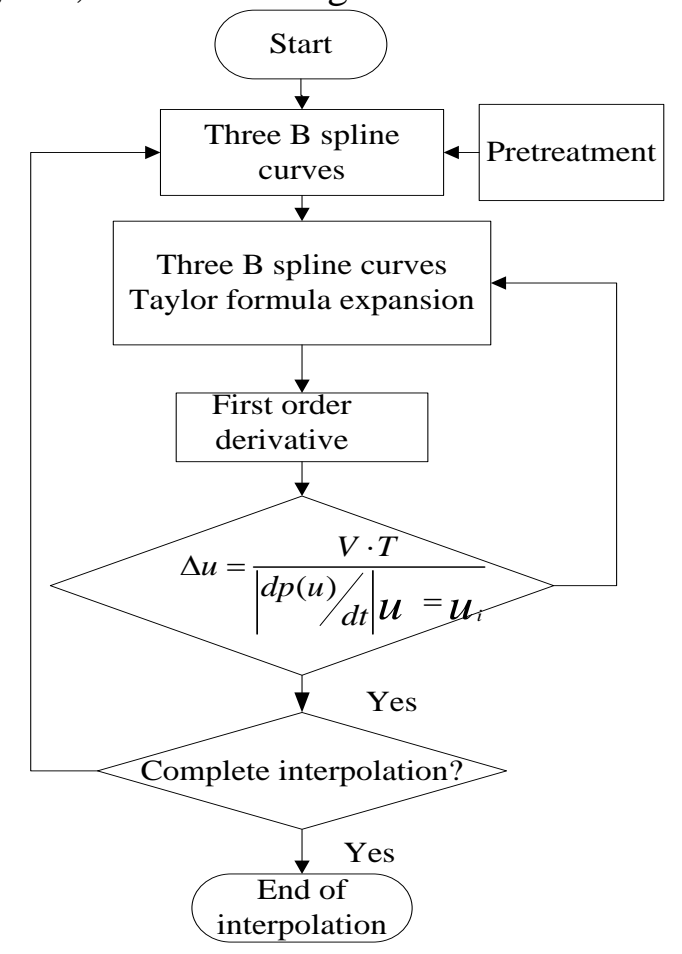

Fig. 1 Flow chart of Three B spline curve interpolation algorithm

Figure 1 for Flow chart of Three B spline curve interpolation algorithm is explained as follow:

Step1: the input date are from Three B spline curve parameters model such as Three B spline curve control points, weight vector and so on.

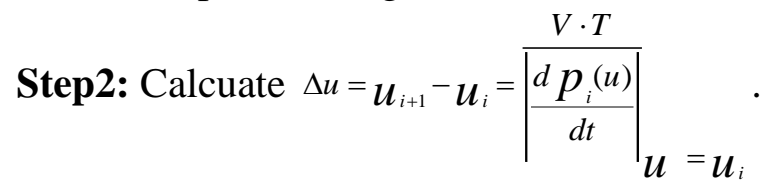

Step3: Get the Three B spline curve position $\left(x_{i}, y_{i}, z_{i}\right)$ can be calculated by using Three B spline curve interpolation algorithm method which exist a $\Delta u$. 
Step4: End of Three B spline curve interpolation.

\section{Experiment simulation and analysis}

\subsection{Interpolation example}

The three B spline curve interpolation system consists five modules: NC code, NURBS interpolation parameters, NURBS interpolation segment, Motion card Controller, B spline curve interpolation is finished, as is shown in Figure 2.

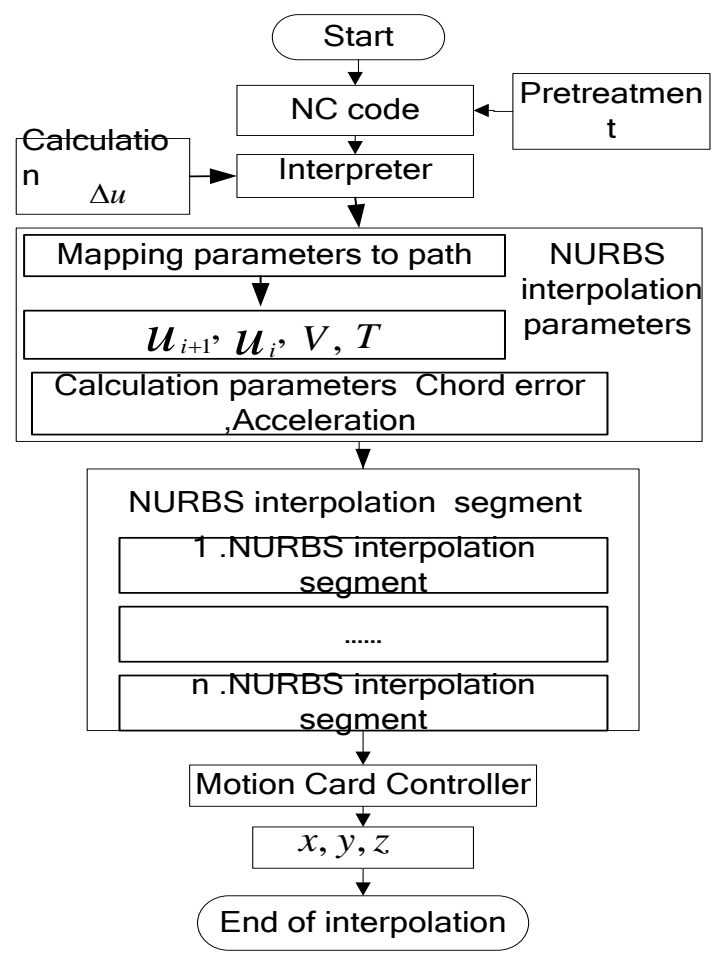

Fig. 2 Three B spline curve interpolation system

The control points, weight vector, and knot vector of this Three B spline curve interpolation are:

The ordinal control points are: $p\left(x_{i}, y_{i}, z_{i}\right)=\left\{\begin{array}{llllllll}1 & 1.1 & 1.3 & 1.5 & 1.5 & 1.6 & 1.7 & 1.8 \\ 1 & 1.1 & 1.2 & 1.5 & 1.5 & 1.7 & 1.8 & 1.9 \\ 5 & 10 & 13 & 14 & 15 & 16 & 17 & 19\end{array}\right\}^{T} \quad i=1,2,3, \ldots(\mathrm{m})$;

The weight vector is: $\mathrm{W}=\{1,100,100,1,100,100,1\}$;

The knot vector is: $\mathrm{U}=\{0,0,0,0.25,0.5,0.5,0.75,1,1,1\}$;

The sampling time in interpolation is: $\mathrm{T}=2 \mathrm{~ms}$;

The feedrate command is: $\mathrm{F}=0.2 \mathrm{~m} / \mathrm{s}$;

The maximal allowable chord error is: $\mathrm{Emax}=1 \mathrm{e}-7 \mathrm{~m}$;

The maximal allowable acceleration/deceleration is: $A m a x=0.5 \mathrm{~m} / \mathrm{s} 2$.

$$
p\left(x_{i}, y_{i}, z_{i}\right)=\left\{\begin{array}{llllllll}
1 & 1.1 & 1.3 & 1.5 & 1.5 & 1.6 & 1.7 & 1.8 \\
1 & 1.1 & 1.2 & 1.5 & 1.5 & 1.7 & 1.8 & 1.9 \\
5 & 10 & 13 & 14 & 15 & 16 & 17 & 19
\end{array}\right\}^{T} \quad i=1,2,3, \cdots
$$

The SIEMENS 810D CNC system has a wide and large share in the CNC system market, and they make NC parameters as NC program command CNC machine tool, Three B spline curve interpolation algorithm based on Three B spline curve G code .G06.2: Three B spline curve.txt, RT ' in the CNC machine tools complete interpolation. By Using computer soft in Three B spline curve interpolation, as shown in Figure 3 and Figure 4.

G06.2 K3 U0 X0 Y0 Z1 W1.6 F18 
U0 X25 Y30 Z6 W1.7

U0 X50 Y50 Z16 W0.7

U0 X65 Y60 Z22 W1.25

U0.6306 X132 Y40 Z41 W1

U0.7426 X142 Y30 Z51 W1.2

U1 X152 Y10 Z21 W1.2

$\mathrm{U} 1$

$\mathrm{U} 1$

U1

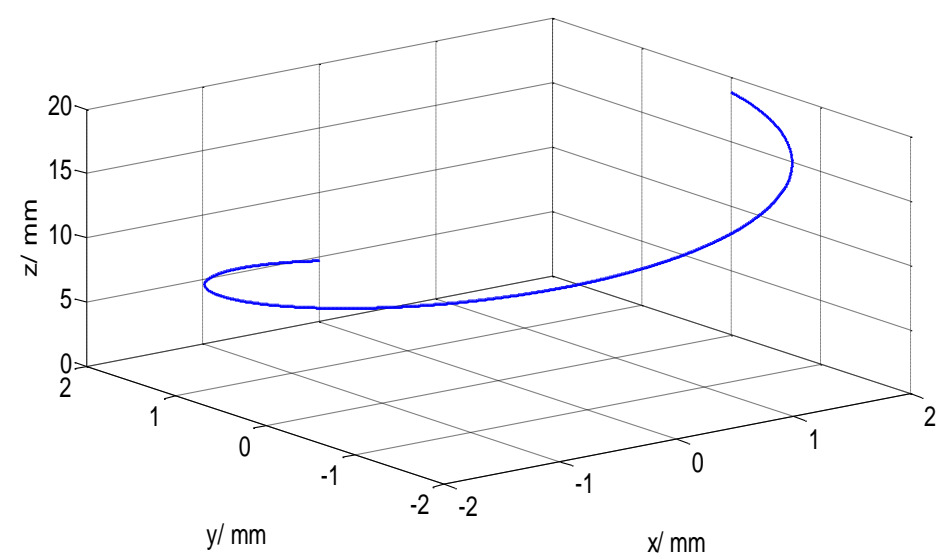

Fig. 3 NURBS curve simulation

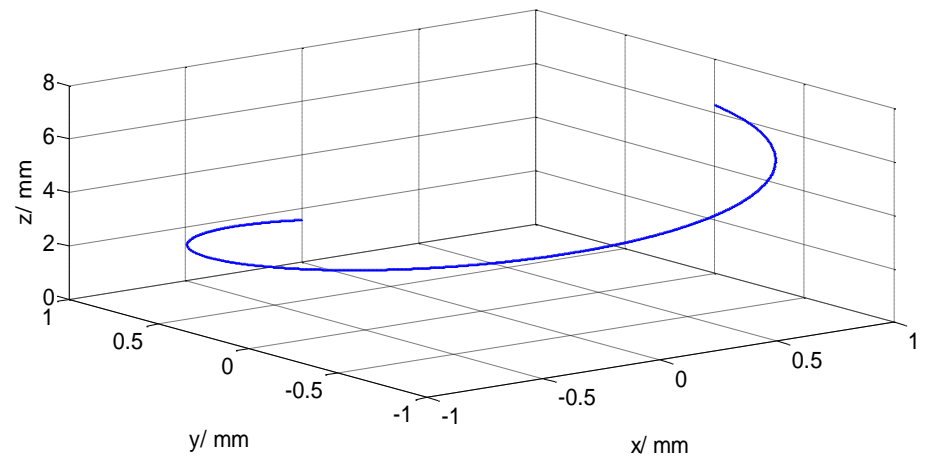

Fig. 4 Three B spline curve interpolation simulation

\subsection{Data analysis}

In order to show the superiority of Three B spline curve interpolation algorithm, the interpolation accuracy and the interpolation time and the interpolation time data table are made according to the graph of Figure 3 and Figure 4, as shown in Table 1.

Tab.1 Table analysis of interpolation of NURBS curve and Three B spline curve interpolation results

\begin{tabular}{|c|c|c|c|}
\hline Parameters algorithms & $\begin{array}{c}\text { Interpolation time } \\
(\mathrm{s})\end{array}$ & $\begin{array}{c}\text { Max step } \\
\text { Error }(\mathrm{mm})\end{array}$ & Min step error (mm) \\
\hline NURBS interpolation & 18.884 & 9.0801 & 0.712 \\
\hline Three B spline curve interpolation & 15.5 & 7.9801 & 0.46 \\
\hline
\end{tabular}

As can be seen from Fig. 3, Fig.4 and able 1, in the process of the NURBS interpolation and Three B spline curve interpolation, interpolation time reduced, Max step error deceased, Max step error value is 7.980 , which meet the expected to interpolation, i.e. to reduce the compensation error and interpolation step chord error. To verify the high efficiency and reliability of this improved three B spline curve interpolation are applied in the experiments to make a comparison. It can be seen that Three B spline curve interpolation is feasible and efficient. 
In a word, the algorithm for Improved three B spline curve interpolation presented in this paper could satisfy high-speed and high-accuracy interpolation requirements, which can be used for actual interpolation processing.

\section{Summary}

Based on Improved three B spline curve interpolation algorithm, Three B spline curve interpolation calculation and general computational complexity, proposed an improved interpolation algorithm, can reduce the time and improve the efficiency of interpolation. The improved method is given by using MATLAB simulation, the results show that the interpolation algorithm can reduce interpolation computation and interpolation operation debugging time and reduce the overshoot, it has strong adaptability and versatility, provides a new method for complex dynamic control of uncertain systems.

\section{Acknowledgements}

The authors thank the financial supports from National Natural Science Foundation of China (Grant no. 51165024) and Science and Technology Major Project of "High-grade NC Machine Tools and Basic Manufacturing Equipment" (2010ZX040001-181).

\section{References}

[1] Fang Yi-Xiang, Liu Wen-Xue. Based on the geometric properties of the cubic uniform B-Spline curve structure description[J]. Journal of engineering graphics, 2 , pp.96-102, 2006.

[2] Zhang Wan-Jun, Hu Chi-Bing, Zhang Feng ,et al . Honing machine motion control card three B spline curve method of interpolation arithmetic for CNC system [J].Chinese Journal of Manufacturing Technology \& Machine Tool , 8(8), pp.40-43,August 2012.

[3] Zhang Wan-Jun, HU Chi-bing, WU Zai-xin, et,al. Research on modification algorithm of Three B Spline curve interpolation technology [J].Chinese Journal of Manufacturing Technology \& Machine Tool ,2 pp.141-143,Feburary 2013.

[4] Zhang Wan-Jun, Zhang Feng, Zhang Guo-hua. Research on a algorithm of adaptive interpolation for NURBS curve. [J].Applied Mechanics and Materials ,Vol. 687-691, pp.1600-1603, December 2014.

[5] Zhang Wan-Jun, Zhang Feng, Zhang Guo-hua. Research on modification algorithm of Cubic B-spline curve interpolation technology. [J].Applied Mechanics and Materials, Vol. 687-691, pp.1596-1599, December 2014.

[6] Zhang Wan-Jun, Zhang Feng, Zhang Wan-Liang. Research on a NURBS curve of timing / interrupt interpolation algorithm for CNC system [J].Chinese Journal of Manufacturing Technology \& Machine Tool , 4(4), pp.183-187,April 2015.

[7] Kong Fan-Guo, Hao Shang-Hua,ZhongYan-Zhi. NURBS curve interpolation algorithm to achieve the VC [J]. China new technology and new products, 17, pp.8-9, 2009.

[8] Ye Bo-Sheng, Yang Shu-Zi. CNC system in cubic B-Spline curve interpolation method [J ]. China Mechanical Engineering, 9 ( 3) , pp. 42 - 43, 1998.

[9] Li He-Cheng, Wang Yu-Ping, An interpolation based genetic algorithm for sloving nonlinear bilevel programming problems.Chinese Journal of Computers, 31(6), pp.910-918, June 2008.

[10] Shpitalni M, Koren Y, Lo CC. Realtime curve interpolators. Computer- Aided Design, 26, pp. 832-838, 1994.

[11] Zhang Wan-Jun, Zhang Feng, Zhang Wan-liang. Research on high-grade CNC machines tools CNC system for B-Spline curve method of High-speed real-time interpolation arithmetic [J]. Chinese Journal of Manufacturing Technology \& Machine Tool , 8(8), pp.172-176,August 2015. 
[12] Huang JT, Yang DCH.A generalized interpolator for command generation of parametric curves in computer controlled machines. Japan/USA Symposium on Flexible Automation, 1(1), pp.3 93-399,January1992.

[13] Lo CC,Chung CY.Curve generation and control for biaxial machine tools. J.CSME ,18, pp.175-182,1997.

[14] Zhang wanjun.A Newton iteration-Rapson of NC interpolation system [P]. China : 2015200430 $82.8,2015$.

[15] Zhang wanjun. Based on b-spline curve real-time interpolation of CNC system at a high speed [P]. China : 201520069002.6,2015.

[16] Zhang wanjun. A kind of based on ARM + FPGA dual CPU of NC interpolation system [P]. China : 201520969163. 0,2016.

[17] Zhang wanjun. A control system of numerical control for interpolation PCI system [P]. China : $201620110057.1,2016$.

[18] Sun, Shujie, Lin, Hu; Zheng, Liaomo. A real-time and look-ahead interpolation methodology with dynamic B-spline transition scheme for CNC machining of short line segments [J]. Internation journal of advanced manufacturing technology, 84 (3) , pp. 1359-1370, 2016.

[19] Duan, Molong, Okwudire, Chinedum. Minimum-time cornering for CNC machines using an optimal control method with NURBSparameterization

[J]. Internation journal of advanced manufacturing technology, 85 (3) , pp. 1405-1418, 2016.

[20]Liu Qiang,Liu Huan, Yuan Songmei.High Accurate Interpolation of NURBS Tool Path for CNC Machine Tools[J]. China journal of mechanical engineering, 29 (5) , pp. 911-920, 2016.

[21] Zhang Liqiang,Zhang, Kai Yan, Yecui.Local Corner Smoothing Transition Algorithm Based on Double Cubic NURBS for Five-axis Linear Tool Path[J].Strojniskivestnik-journal of mechanical engineering, 29 (5), pp. 647-656, 2016(9). 